\title{
Growth Optimal Investment with Transaction Costs $^{\star}$
}

\author{
László Györfi and István Vajda \\ Department of Computer Science and Information Theory, \\ Budapest University of Technology and Economics, \\ Magyar Tudósok Körútja 2., Budapest, Hungary, H-1117 \\ \{gyorfi, vajda\}@szit.bme.hu
}

\begin{abstract}
Discrete time infinite horizon growth optimal investment in stock markets with transactions costs is considered. The stock processes are modelled by homogeneous Markov processes. Assuming that the distribution of the market process is known, we show two recursive investment strategies such that, in the long run, the growth rate on trajectories (in "liminf" sense) is greater than or equal to the growth rate of any other investment strategy with probability 1 .
\end{abstract}

\section{Introduction}

The purpose of this paper is to investigate sequential investment strategies for financial markets such that the strategies are allowed to use information collected from the past of the market and determine, at the beginning of a trading period, a portfolio, that is, a way to distribute their current capital among the available assets. The goal of the investor is to maximize his wealth on the long run. If there is no transaction cost and the price relatives form a stationary and ergodic process the best strategy (called log-optimum strategy) can be constructed in full knowledge of the distribution of the entire process, see Algoet and Cover [2].

Papers dealing with growth optimal investment with transaction costs in discrete time setting are seldom. Cover and Iyengar [13] formulated the problem of horse race markets, where in every market period one of the assets has positive pay off and all the others pay nothing. Their model included proportional transaction costs and they used a long run expected average reward criterion. There are results for more general markets as well. Iyengar [12 investigated growth optimal investment with several assets assuming independent and identically distributed (i.i.d.) sequence of asset returns. Bobryk and Stettner 4 considered the case of portfolio selection with consumption, when there are two assets, a bank account and a stock. Furthermore, long run expected discounted reward and i.i.d asset returns were assumed. In the case of discrete time, the most far reaching study was Schäfer [16] who considered the maximization of the long run expected growth rate with several assets and proportional transaction costs,

\footnotetext{
* This research was supported by the Computer and Automation Research Institute of the Hungarian Academy of Sciences.
} 
when the asset returns follow a stationary Markov process. In contrast to the previous literature we assume only that the stock processes are modelled by homogeneous (there is no requirement regarding the initial distribution) Markov processes. We extend the usual framework of analyzing expected growth rate by showing two recursive investment strategies such that, in the long run, the growth rate on trajectories is greater than or equal to the growth rate of any other investment strategy with probability 1 .

The rest of the paper is organized as follows. In Section 2 we introduce the market model and describe the modelling of transaction costs. In Section 3 we formulate the underlying Markov control problem, and Section 4 defines optimal portfolio selection strategies. Following the Summary in Section 5, the proofs are given in Section 6 .

\section{Mathematical Setup: Investment with Transaction Cost}

Consider a market consisting of $d$ assets. The evolution of the market in time is represented by a sequence of market vectors $\mathbf{s}_{1}, \mathbf{s}_{2}, \ldots \in \mathbb{R}_{+}^{d}$, where $\mathbf{s}_{i}=$ $\left(s_{i}^{(1)}, \ldots, s_{i}^{(d)}\right)$ such that the $j$-th component $s_{i}^{(j)}$ of $\mathbf{s}_{i}$ denotes the price of the $j$-th asset at the end of the $i$-th trading period. $\left(s_{0}^{(j)}=1\right.$.)

In order to apply the usual prediction techniques for time series analysis one has to transform the sequence $\left\{\mathbf{s}_{i}\right\}$ into a more or less stationary sequence of return vectors $\left\{\mathbf{x}_{i}\right\}$ as follows: $\mathbf{x}_{i}=\left(x_{i}^{(1)}, \ldots, x_{i}^{(d)}\right)$ such that $x_{i}^{(j)}=\frac{s_{i}^{(j)}}{s_{i-1}^{(j)}}$. Thus, the $j$-th component $x_{i}^{(j)}$ of the return vector $\mathbf{x}_{i}$ denotes the amount obtained after investing a unit capital in the $j$-th asset on the $i$-th trading period.

The investor is allowed to diversify his capital at the beginning of each trading period according to a portfolio vector $\mathbf{b}=\left(b^{(1)}, \ldots b^{(d)}\right)^{T}$. The $j$-th component $b^{(j)}$ of $\mathbf{b}$ denotes the proportion of the investor's capital invested in asset $j$. Throughout the paper we assume that the portfolio vector $\mathbf{b}$ has nonnegative components with $\sum_{j=1}^{d} b^{(j)}=1$. The fact that $\sum_{j=1}^{d} b^{(j)}=1$ means that the investment strategy is self financing and consumption of capital is excluded. The non-negativity of the components of $\mathbf{b}$ means that short selling and buying stocks on margin are not permitted. To make the analysis feasible, some simplifying assumptions are used that need to be taken into account. We assume that assets are arbitrarily divisible and all assets are available in unbounded quantities at the current price at any given trading period. We also assume that the behavior of the market is not affected by the actions of the investor using the strategies under investigation.

For $j \leq i$ we abbreviate by $\mathbf{x}_{j}^{i}$ the array of return vectors $\left(\mathbf{x}_{j}, \ldots, \mathbf{x}_{i}\right)$. Denote by $\Delta_{d}$ the simplex of all vectors $\mathbf{b} \in \mathbb{R}_{+}^{d}$ with nonnegative components summing up to one. An investment strategy is a sequence $\mathbf{B}$ of functions

$$
\mathbf{b}_{i}:\left(\mathbb{R}_{+}^{d}\right)^{i-1} \rightarrow \Delta_{d}, \quad i=1,2, \ldots
$$

so that $\mathbf{b}_{i}\left(\mathbf{x}_{1}^{i-1}\right)$ denotes the portfolio vector chosen by the investor on the $i$ th trading period, upon observing the past behavior of the market. We write $\mathbf{b}\left(\mathbf{x}_{1}^{i-1}\right)=\mathbf{b}_{i}\left(\mathbf{x}_{1}^{i-1}\right)$ to ease the notation. 
Let $S_{n}$ denote the gross wealth at the end of trading period $n, n=0,1,2, \cdots$, where without loss of generality let the investor's initial capital $S_{0}$ be 1 dollar, while $N_{n}$ stands for the net wealth at the end of trading period $n$. Using the above notations, for the trading period $n$, the net wealth $N_{n-1}$ can be invested according to the portfolio $\mathbf{b}_{n}$, therefore the gross wealth $S_{n}$ at the end of trading period $n$ is

$$
S_{n}=N_{n-1} \sum_{j=1}^{d} b_{n}^{(j)} x_{n}^{(j)}=N_{n-1}\left\langle\mathbf{b}_{n}, \mathbf{x}_{n}\right\rangle,
$$

where $\langle\cdot, \cdot\rangle$ denotes inner product.

At the beginning of a new market day $n+1$, the investor sets up his new portfolio, i.e. buys/sells stocks according to the actual portfolio vector $\mathbf{b}_{n+1}$. During this rearrangement, he has to pay transaction cost, therefore at the beginning of a new market day $n+1$ the net wealth $N_{n}$ in the portfolio $\mathbf{b}_{n+1}$ is less than $S_{n}$. The rate of proportional transaction costs (commission factors) levied on one asset are denoted by $0<c_{s}<1$ and $0<c_{p}<1$, i.e., the sale of 1 dollar worth of asset $i$ nets only $1-c_{s}$ dollars, and similarly we take into account the purchase of an asset such that the purchase of 1 dollar's worth of asset $i$ costs an extra $c_{p}$ dollars. We consider the special case when the rate of costs are constant over the assets. Let's calculate the transaction cost to be paid when select the portfolio $\mathbf{b}_{n+1}$. Before rearranging the capitals, at the $j$-th asset there are $b_{n}^{(j)} x_{n}^{(j)} N_{n-1}$ dollars, while after rearranging we need $b_{n+1}^{(j)} N_{n}$ dollars. If $b_{n}^{(j)} x_{n}^{(j)} N_{n-1} \geq b_{n+1}^{(j)} N_{n}$ then we have to sell and $c_{s}\left(b_{n}^{(j)} x_{n}^{(j)} N_{n-1}-b_{n+1}^{(j)} N_{n}\right)$ is the transaction cost at the $j$-th asset is, otherwise we have to buy and $c_{p}\left(b_{n+1}^{(j)} N_{n}-b_{n}^{(j)} x_{n}^{(j)} N_{n-1}\right)$ is the transaction cost at the $j$-th asset.

Let $x^{+}$denote the positive part of $x$. Thus, the gross wealth $S_{n}$ decomposes to the sum of the net wealth and cost the following - self-financing - way $S_{n}=$ $N_{n}+c_{s} \sum_{j=1}^{d}\left(b_{n}^{(j)} x_{n}^{(j)} N_{n-1}-b_{n+1}^{(j)} N_{n}\right)^{+}+c_{p} \sum_{j=1}^{d}\left(b_{n+1}^{(j)} N_{n}-b_{n}^{(j)} x_{n}^{(j)} N_{n-1}\right)^{+}$.

Dividing both sides by $S_{n}$ and introducing the ratio $w_{n}=\frac{N_{n}}{S_{n}}, 0<w_{n}<1$, we get

$$
1=w_{n}+c_{s} \sum_{j=1}^{d}\left(\frac{b_{n}^{(j)} x_{n}^{(j)}}{\left\langle\mathbf{b}_{n}, \mathbf{x}_{n}\right\rangle}-b_{n+1}^{(j)} w_{n}\right)^{+}+c_{p} \sum_{j=1}^{d}\left(b_{n+1}^{(j)} w_{n}-\frac{b_{n}^{(j)} x_{n}^{(j)}}{\left\langle\mathbf{b}_{n}, \mathbf{x}_{n}\right\rangle}\right)^{+}
$$

Remark 1. Equation (1) is used in the sequel. Examining this cost equation, it turns out, that for arbitrary portfolio vectors $\mathbf{b}_{n}, \mathbf{b}_{n+1}$, and return vector $\mathbf{x}_{n}$ there exists a unique cost factor $w_{n} \in[0,1)$, i.e. the portfolio is self financing. The value of cost factor $w_{n}$ at day $n$ is determined by portfolio vectors $\mathbf{b}_{n}$ and $\mathbf{b}_{n+1}$ as well as by return vector $\mathbf{x}_{n}$, i.e. $w_{n}=w\left(\mathbf{b}_{n}, \mathbf{b}_{n+1}, \mathbf{x}_{n}\right)$, for some function $w$. If we want to rearrange our portfolio substantially, then our net wealth decreases more considerably, however, it remains positive. Note also, that the cost does not restrict the set of new portfolio vectors, i.e., the optimization algorithm searches for optimal vector $\mathbf{b}_{n+1}$ within the whole simplex $\Delta_{d}$. The value of the cost factor ranges between $\frac{1-c_{s}}{1+c_{p}} \leq w_{n} \leq 1$. 
Starting with an initial wealth $S_{0}=1$ and $w_{0}=1$, wealth $S_{n}$ at the closing time of the $n$-th market day becomes $S_{n}=N_{n-1}\left\langle\mathbf{b}_{n}, \mathbf{x}_{n}\right\rangle=w_{n-1} S_{n-1}\left\langle\mathbf{b}_{n}, \mathbf{x}_{n}\right\rangle$ $=\prod_{i=1}^{n}\left[w\left(\mathbf{b}_{i-1}, \mathbf{b}_{i}, \mathbf{x}_{i-1}\right)\left\langle\mathbf{b}_{i}, \mathbf{x}_{i}\right\rangle\right]$. Introduce the notation

$$
g\left(\mathbf{b}_{i-1}, \mathbf{b}_{i}, \mathbf{x}_{i-1}, \mathbf{x}_{i}\right)=\log \left(w\left(\mathbf{b}_{i-1}, \mathbf{b}_{i}, \mathbf{x}_{i-1}\right)\left\langle\mathbf{b}_{i}, \mathbf{x}_{i}\right\rangle\right)
$$

then the average growth rate becomes

$$
\frac{1}{n} \log S_{n}=\frac{1}{n} \sum_{i=1}^{n} \log \left(w\left(\mathbf{b}_{i-1}, \mathbf{b}_{i}, \mathbf{x}_{i-1}\right)\left\langle\mathbf{b}_{i}, \mathbf{x}_{i}\right\rangle\right)=\frac{1}{n} \sum_{i=1}^{n} g\left(\mathbf{b}_{i-1}, \mathbf{b}_{i}, \mathbf{x}_{i-1}, \mathbf{x}_{i}\right)(3)
$$

Our aim is to maximize this average growth rate.

In the sequel $\mathbf{x}_{i}$ will be random variable and is denoted by $\mathbf{X}_{i}$. Let's use the decomposition

$$
\frac{1}{n} \log S_{n}=I_{n}+J_{n},
$$

where $I_{n}$ is $\frac{1}{n} \sum_{i=1}^{n}\left(g\left(\mathbf{b}_{i-1}, \mathbf{b}_{i}, \mathbf{X}_{i-1}, \mathbf{X}_{i}\right)-\mathbb{E}\left\{g\left(\mathbf{b}_{i-1}, \mathbf{b}_{i}, \mathbf{X}_{i-1}, \mathbf{X}_{i}\right) \mid \mathbf{X}_{1}^{i-1}\right\}\right)$ and $J_{n}=\frac{1}{n} \sum_{i=1}^{n} \mathbb{E}\left\{g\left(\mathbf{b}_{i-1}, \mathbf{b}_{i}, \mathbf{X}_{i-1}, \mathbf{X}_{i}\right) \mid \mathbf{X}_{1}^{i-1}\right\} . I_{n}$ is an average of martingale differences. Under mild conditions on the support of the distribution of $\mathbf{X}$, $g\left(\mathbf{b}_{i-1}, \mathbf{b}_{i}, \mathbf{X}_{i-1}, \mathbf{X}_{i}\right)$ is bounded, therefore $I_{n}$ is an average of bounded martingale differences, which converges to 0 almost surely, since according to the Chow Theorem (cf. Theorem 3.3.1 in Stout [18]) $\sum_{i=1}^{\infty} \frac{\mathbb{E}\left\{g\left(\mathbf{b}_{i-1}, \mathbf{b}_{i}, \mathbf{X}_{i-1}, \mathbf{X}_{i}\right)^{2}\right\}}{i^{2}}<\infty$ implies that $I_{n} \rightarrow 0$ almost surely. Thus, the asymptotic maximization of the average growth rate $\frac{1}{n} \log S_{n}$ is equivalent to the maximization of $J_{n}$.

If the market process $\left\{\mathbf{X}_{i}\right\}$ is a homogeneous and first order Markov process then, for appropriate portfolio selection $\left\{\mathbf{b}_{i}\right\}$, we have that

$$
\begin{aligned}
& \mathbb{E}\left\{g\left(\mathbf{b}_{i-1}, \mathbf{b}_{i}, \mathbf{X}_{i-1}, \mathbf{X}_{i}\right) \mid \mathbf{X}_{1}^{i-1}\right\} \\
= & \mathbb{E}\left\{\log \left(w\left(\mathbf{b}_{i-1}, \mathbf{b}_{i}, \mathbf{X}_{i-1}\right)\left\langle\mathbf{b}_{i}, \mathbf{X}_{i}\right\rangle\right) \mid \mathbf{X}_{1}^{i-1}\right\} \\
= & \log w\left(\mathbf{b}_{i-1}, \mathbf{b}_{i}, \mathbf{X}_{i-1}\right)+\mathbb{E}\left\{\log \left\langle\mathbf{b}_{i}, \mathbf{X}_{i}\right\rangle \mid \mathbf{X}_{1}^{i-1}\right\} \\
= & \log w\left(\mathbf{b}_{i-1}, \mathbf{b}_{i}, \mathbf{X}_{i-1}\right)+\mathbb{E}\left\{\log \left\langle\mathbf{b}_{i}, \mathbf{X}_{i}\right\rangle \mid \mathbf{b}_{i}, \mathbf{X}_{i-1}\right\} \\
\stackrel{\text { def }}{=} & v\left(\mathbf{b}_{i-1}, \mathbf{b}_{i}, \mathbf{X}_{i-1}\right),
\end{aligned}
$$

therefore the maximization of the average growth rate $\frac{1}{n} \log S_{n}$ is asymptotically equivalent to the maximization of

$$
J_{n}=\frac{1}{n} \sum_{i=1}^{n} v\left(\mathbf{b}_{i-1}, \mathbf{b}_{i}, \mathbf{X}_{i-1}\right)
$$

Remark 2. Without transaction cost, the fundamental limits, determined in Algoet and Cover [2] reveal that the so-called log-optimum portfolio $\mathbf{B}^{*}=\left\{\mathbf{b}^{*}(\cdot)\right\}$ is the best possible choice. More precisely, in trading period $n$ let $\mathbf{b}^{*}(\cdot)$ be such that $\mathbf{b}_{n}^{*}\left(\mathbf{X}_{1}^{n-1}\right)=\arg \max _{\mathbf{b}(\cdot)} \mathbb{E}\left\{\log \left\langle\mathbf{b}\left(\mathbf{X}_{1}^{n-1}\right), \mathbf{X}_{n}\right\rangle \mid \mathbf{X}_{1}^{n-1}\right\}$. If $S_{n}^{*}=S_{n}\left(\mathbf{B}^{*}\right)$ denotes the capital achieved by a log-optimum portfolio strategy $\mathbf{B}^{*}$, after $n$ trading periods, then for any other investment strategy B with capital $S_{n}=S_{n}(\mathbf{B})$ and 
for any stationary and ergodic return process $\left\{\mathbf{X}_{n}\right\}_{-\infty}^{\infty}, \liminf _{n \rightarrow \infty} \frac{1}{n} \log \frac{S_{n}^{*}}{S_{n}} \geq$ 0 almost surely. Note that for first order Markovian return process $\mathbf{b}_{n}^{*}\left(\mathbf{X}_{1}^{n-1}\right)=$ $\mathbf{b}_{n}^{*}\left(\mathbf{X}_{n-1}\right)=\arg \max _{\mathbf{b}(\cdot)} \mathbb{E}\left\{\log \left\langle\mathbf{b}\left(\mathbf{X}_{n-1}\right), \mathbf{X}_{n}\right\rangle \mid \mathbf{X}_{n-1}\right\}$.

Before introducing to optimal strategies we show to empirical suboptimal algorithms with some experimental results.

Algorithm 1. For transaction cost, one may apply the portfolio $\mathbf{b}_{n}^{*}\left(\mathbf{X}_{n-1}\right)$, and after calculating the portfolio subtract the transaction cost. Let's consider it's empirical counterpart in case of unknown market process. Apply the kernel based log-optimal portfolio selection introduced by Györfi, Lugosi and Udina [8] as follows: define an infinite array of experts $\mathbf{B}^{(\ell)}=\left\{\mathbf{b}^{(\ell)}(\cdot)\right\}$, where $\ell$ is a positive integer. For fixed positive integer $\ell$, choose the radius $r_{\ell}>0$ such that $\lim _{\ell \rightarrow \infty} r_{\ell}=0$. Then, for $n>1$, define the expert $\mathbf{b}^{(\ell)}$ as follows. Put

$$
\mathbf{b}_{n}^{(\ell)}=\underset{\mathbf{b} \in \Delta_{d}}{\arg \max } \sum_{\left\{i<n:\left\|\mathbf{x}_{i-1}-\mathbf{x}_{n-1}\right\| \leq r_{\ell}\right\}} \ln \left\langle\mathbf{b}, \mathbf{x}_{i}\right\rangle,
$$

if the sum is non-void, and $\mathbf{b}_{0}=(1 / d, \ldots, 1 / d)$ otherwise, where $\|\cdot\|$ denotes the Euclidean norm. These experts are aggregated as follows: let $\left\{q_{\ell}\right\}$ be a probability distribution over the set of all positive integers $\ell$. Consider two types of aggregations: By aggregating with the wealth the initial capital $S_{0}=1$ is distributed among the expert according to the distribution $\left\{q_{\ell}\right\}$, and the expert makes the portfolio selection and pays for transaction cost individually. If $S_{n}\left(\mathbf{B}^{(\ell)}\right)$ is the capital accumulated by the elementary strategy $\mathbf{B}^{(\ell)}$ the investor's wealth after period $n$, aggregations $S_{n}=\sum_{\ell} q_{\ell} S_{n}\left(\mathbf{B}^{(\ell)}\right)$. In the second case the aggregated portfolio pays for transaction cost. Here $S_{n}\left(\mathbf{B}^{(\ell)}\right)$ is again the capital accumulated by the elementary strategy $\mathbf{B}^{(\ell)}$ after $n$ periods. Then, after period $n$, the investor's aggregated portfolio becomes $\mathbf{b}_{n}=\frac{\sum_{\ell} q_{\ell} S_{n-1}\left(\mathbf{B}^{(\ell)}\right) \mathbf{b}_{n}^{(\ell)}}{\sum_{\ell} q_{\ell} S_{n-1}\left(\mathbf{B}^{(\ell)}\right)}$. The investor's capital is $S_{n}=S_{n-1}\left\langle\mathbf{b}_{n}, \mathbf{x}_{n}\right\rangle w\left(\mathbf{b}_{n-1}, \mathbf{b}_{n}, \mathbf{x}_{n-1}\right)$, so the aggregated portfolio pays for the transaction cost.

Algorithm 2. Our second suboptimal strategy is a one-step optimization as follows: put $\mathbf{b}_{1}=\{1 / d, \ldots, 1 / d\}$ and for $i \geq 1, \mathbf{b}_{i+1}=\arg \max _{\mathbf{b}^{\prime}} v\left(\mathbf{b}_{i}, \mathbf{b}^{\prime}, \mathbf{X}_{i}\right)$. Obviously, this portfolio has no global optimality property. Let's consider its empirical counterpart in case of unknown distribution. Put $\mathbf{b}_{1}=\{1 / d, \ldots, 1 / d\}$ and for $n \geq 1$,

$$
\mathbf{b}_{n}^{(\ell)}=\underset{\mathbf{b} \in \Delta_{d}}{\arg \max } \sum_{\left\{i<n:\left\|\mathbf{x}_{i-1}-\mathbf{x}_{n-1}\right\| \leq r_{\ell}\right\}}\left(\ln \left\langle\mathbf{b}, \mathbf{x}_{i}\right\rangle+\ln w\left(\mathbf{b}_{n-1}, \mathbf{b}, \mathbf{x}_{n-1}\right)\right),
$$

if the sum is non-void, and $\mathbf{b}_{0}=(1 / d, \ldots, 1 / d)$ otherwise. These elementary portfolios are mixed as in Algortihm1.

The investment strategies are tested on a data set containing 23 stocks and has length 44 years - 11178 trading days ending in 2006 - achievable at www.szit.bme.hu /oti/portfolio. The proposed empirical portfolio selection algorithms use an infinite set of experts. In the experiment we selected $L=10$. Choose $\left\{q_{\ell}\right\}=1 / L$ over the experts in use, and the radius $r_{\ell}^{2}=0.0001 \cdot d \cdot \ell, r_{\ell}^{2}=0.0002 \cdot d+$ 
$0.00002 \cdot d \cdot \ell, \quad$ for $\ell=1, \ldots, L$. Table [1] [10 summarizes the average annual yield achieved by each expert at the last period when investing one unit for the kernel-based log-optimal portfolio. Experts are indexed by $\ell=1 \ldots 10$ in rows. The second column contains the average annual yields of experts for kernel based log-optimal portfolio if there is no transaction cost, and in this case the results of the two aggregations are the same: $124 \%$. The third and fourth columns contain the average annual yields of experts for kernel based log-optimal portfolio if the commission factor is $c=0.0015$.

In the sequel we prove to optimal strategies in case of known distribution as these algorithms were not optimal. The empirical optimal counterparts of them are still missing.

\section{The Related Markov Control Problem}

The problem of optimal investment with proportional transaction costs has been essentially formulated in continuous time only, in the classical articles Davis and Norman [6], Taksar et al. [19] and Shreve and Soner [17], etc. Taksar, Klass and Assaf [19] investigate optimal investment in a continuous time market with two assets and with proportional transaction costs driven by a Wiener process and using long run expected reward criteria. Akien, Sulem and Taksar [1] extend these results to the case of several risky assets.

Most of the above mentioned papers use some kind of method from stochastic optimal control theory. Without exception all the papers consider optimality in expected reward. None of these papers give result on almost sure optimality. In this paper we present two portfolio selection strategies, and for a Markovian market we prove their almost sure optimality.

Discrete time portfolio optimization with transaction cost is a special case of the general Markov control processes (MCP). A discrete time Markov control process

Table 1. The average annual yields of the individual experts and of the aggregations with $c=0.0015$

\begin{tabular}{|r|r|r|r|}
\hline$\ell$ & $c=0$ & Algorithm 1 & Algorithm 2 \\
\hline 1 & $68 \%$ & $-6 \%$ & $27 \%$ \\
\hline 2 & $87 \%$ & $3 \%$ & $38 \%$ \\
\hline 3 & $94 \%$ & $7 \%$ & $45 \%$ \\
\hline 4 & $94 \%$ & $6 \%$ & $49 \%$ \\
\hline 5 & $108 \%$ & $14 \%$ & $54 \%$ \\
\hline 6 & $118 \%$ & $19 \%$ & $60 \%$ \\
\hline 7 & $122 \%$ & $21 \%$ & $69 \%$ \\
\hline 8 & $128 \%$ & $25 \%$ & $73 \%$ \\
\hline 9 & $131 \%$ & $27 \%$ & $71 \%$ \\
\hline 10 & $131 \%$ & $28 \%$ & $72 \%$ \\
\hline Aggregation with wealth & $124 \%$ & $\mathbf{2 4 \%}$ & $\mathbf{6 8 \%}$ \\
\hline Aggregation with portfolio & $124 \%$ & $\mathbf{2 9 \%}$ & $\mathbf{7 3 \%}$ \\
\hline
\end{tabular}


is defined by a five tuple $(S, A, U(s), Q, r)$ (cf. [11]). $S$ is a Borel space, called the state space, the action space $A$ is Borel, too, the space of admissible actions $U(s)$ is a Borel subset of $A$. Let the set $K$ be $\{(s, a): s \in S, a \in U(s)\}$. The transition law is a stochastic kernel $Q(. \mid s, a)$ on $S$ given $K$, and $r(s, a)$ is the reward function.

The evolution of the process is the following. Let $S_{t}$ denote the the state at time t, action $A_{t}$ is chosen at that time. Let $S_{t}=s$ and $A_{t}=a$, then the reward is $r(s, a)$, and the process moves to $S_{t+1}$ according to the kernel $Q(. \mid s, a)$. A control policy is a sequence $\pi=\left\{\pi_{n}\right\}$ of deterministic functions, which can also be stochastic kernels on $A$ given the past states and actions.

Two reward criteria are considered. The expected long run average reward for $\pi$ is defined by $J(\pi)=\liminf _{n \rightarrow \infty} \frac{1}{n} \sum_{t=0}^{n-1} \mathbb{E}^{\pi} r\left(S_{t}, A_{t}\right)$. The sample-path average reward is defined as $J(\pi):=\liminf _{n \rightarrow \infty} \frac{1}{n} \sum_{t=0}^{n-1} r\left(S_{t}, A_{t}\right)$. In the theory of MCP most of the results correspond to expected long run average reward, while just a few present result for the sample-path criterion. Such sample-path results can be found in [3] for bounded rewards and in [20], [14] for unbounded rewards. For Markov control processes, the main aim is to approach the maximum asymptotic reward: $J^{*}=\sup _{\pi} J(\pi)$, which leads to a dynamic programming problem.

For portfolio optimization with transaction cost, we formulate the corresponding Markov control problem. Assume that there exist $0<a_{1}<1<a_{2}<\infty$ such that

$$
\mathbf{X}_{i} \in\left[a_{1}, a_{2}\right]^{d} \text {. }
$$

1. Let us define the state space as: $S:=\left\{(\mathbf{b}, \mathbf{x}) \mid \mathbf{b} \in \Delta_{d}, \mathbf{x} \in\left[a_{1}, a_{2}\right]^{d}\right\}$.

2. The action space is $A:=\Delta_{d}$.

3. For the set of admissible actions we get $U(\mathbf{b}, \mathbf{x}):=\Delta_{d}$.

4. The stochastic kernel is $Q\left(d\left(\mathbf{b}^{\prime}, \mathbf{x}^{\prime}\right) \mid(\mathbf{b}, \mathbf{x}), \mathbf{b}^{\prime}\right):=P\left(d \mathbf{x}^{\prime} \mid \mathbf{x}\right):=\mathbb{P}\left\{d \mathbf{X}_{2}=\right.$ $\left.d \mathbf{x}^{\prime} \mid \mathbf{X}_{1}=\mathbf{x}\right\}$ the transition probability distribution of the Markov market process, describing the asset returns. Note, that this corresponds to the assumption, that the market behaviour is not affected by the investor.

5 . The reward function is: $r\left((\mathbf{b}, \mathbf{x}), \mathbf{b}^{\prime}\right)=v\left(\mathbf{b}, \mathbf{b}^{\prime}, \mathbf{x}\right)$.

6. The sample-path average reward criterion is the following:

$\liminf _{n \rightarrow \infty} \frac{1}{n} \sum_{t=1}^{n} r\left(\left(\mathbf{b}_{t-1}, \mathbf{x}_{t-1}\right), \mathbf{b}_{t}\right)=\liminf _{n \rightarrow \infty} \frac{1}{n} \sum_{t=1}^{n} v\left(\mathbf{b}_{t-1}, \mathbf{b}_{t}, \mathbf{x}_{t-1}\right)$ $=\liminf _{n \rightarrow \infty} J_{n}$.

Remark 3. It should be noted that the methods of MCP literature, more precisely the theorems in [3], 20], [14] can't be applied in our case. However, we do use the formalism, the results on the existence of the solution of discounted Bellman equations, and the basic idea of vanishing discount approach.

\section{Optimal Portfolio Selection Algorithms}

We introduce two optimal portfolio selection strategies. Let $0<\delta<1$ denote a discount factor. We apply a kind of vanishing discount approach, formulated by the discounted Bellman equation:

$$
F_{\delta}(\mathbf{b}, \mathbf{x})=\max _{\mathbf{b}^{\prime}}\left\{v\left(\mathbf{b}, \mathbf{b}^{\prime}, \mathbf{x}\right)+(1-\delta) \mathbb{E}\left\{F_{\delta}\left(\mathbf{b}^{\prime}, \mathbf{X}_{2}\right) \mid \mathbf{X}_{1}=\mathbf{x}\right\}\right\} .
$$


It can be shown that this discounted Bellman equation has a solution (cf. Hernández-Lerma, Lasserie [11, Schäfer [16]).

Strategy 1 . Our first portfolio selection strategy is the following put $\mathbf{b}_{1}^{*}=$ $\{1 / d, \ldots, 1 / d\}$ and

$$
\mathbf{b}_{i+1}^{*}=\underset{\mathbf{b}^{\prime}}{\arg \max }\left\{v\left(\mathbf{b}_{i}^{*}, \mathbf{b}^{\prime}, \mathbf{X}_{i}\right)+\left(1-\delta_{i}\right) \mathbb{E}\left\{F_{\delta_{i}}\left(\mathbf{b}^{\prime}, \mathbf{X}_{i+1}\right) \mid \mathbf{X}_{i}\right\}\right\},
$$

for $1 \leq i$, where $0<\delta_{i}<1$ is a discount factor such that $\delta_{i} \downarrow 0$.

Remark 4. A strategy similar to (9) was defined by Schäfer [16]. He introduces an additional asset to settle the transaction costs when the portfolio is restructured.

Remark 5. A portfolio selection $\left\{\mathbf{b}_{i}\right\}$ is called recursive if it has the form $\mathbf{b}_{i}=$ $\mathbf{b}_{i}\left(\mathbf{x}_{1}^{i-1}\right)=\mathbf{b}_{i}\left(\mathbf{b}_{i-1}, \mathbf{x}_{i-1}\right)$. Obviously, the portfolio $\left\{\mathbf{b}_{i}^{*}\right\}$ is recursive. The recursion in the definition of the portfolio $\left\{\mathbf{b}_{i}^{*}\right\}$ is not time invariant, i.e., it is a non-stationary portfolio selection rule.

Now, we claim our result on the optimality of Strategy 1 with respect to a sample-path average criterion:

Theorem 1. Assume

(i) that $\left\{\mathbf{X}_{i}\right\}$ is a homogeneous and first order Markov process,

(ii) and there exist $0<a_{1}<1<a_{2}<\infty$ such that $a_{1} \leq X^{(j)} \leq a_{2}$ for all $j=1, \ldots, d$.

Choose the discount factor $\delta_{i} \downarrow 0$ such that $\left(\delta_{i}-\delta_{i+1}\right) / \delta_{i+1}^{2} \rightarrow 0$ as $i \rightarrow \infty$, and $\sum_{n=1}^{\infty} \frac{1}{n^{2} \delta_{n}^{2}}<\infty$. Then, for Strategy 1 , the portfolio $\left\{\mathbf{b}_{i}^{*}\right\}$ with capital $S_{n}^{*}$ is optimal in the sense that for any portfolio strategy $\left\{\mathbf{b}_{i}\right\}$ with capital $S_{n}$,

$$
\liminf _{n \rightarrow \infty}\left(\frac{1}{n} \log S_{n}^{*}-\frac{1}{n} \log S_{n}\right) \geq 0 \text { a.s. }
$$

Remark 6. $\liminf \operatorname{in}_{n \rightarrow \infty} \mathbb{E}\left\{\frac{1}{n} \log S_{n}^{*}-\frac{1}{n} \log S_{n}\right\} \geq 0$, according to Theorem 4.2.1 in Schäfer [16], i.e., the portfolio $\left\{\mathbf{b}_{i}^{*}\right\}$ is optimal in expectation. Theorem 1 states that the portfolio strategy $\left\{\mathbf{b}_{i}^{*}\right\}$ is sample-path optimal, too, i.e., it is optimal with probability one.

Remark \%. For the choice $\delta_{i}=i^{-\epsilon}$, with $\epsilon<1 / 2$, the conditions of Theorem 1 are satisfied.

Remark 8. For the standard stock market problems, the condition (i) is satisfied with $a_{1}=0.9 \quad$ and $a_{2}=1.1$, (cf.Fernholz [7]).

Strategy 2. Next, we introduce a portfolio with stationary (time invariant) recursion such that this portfolio is a sample-path optimal policy, too. For any integer $1 \leq k$, put $\mathbf{b}_{1}^{(k)}=\{1 / d, \ldots, 1 / d\}$ and

$$
\mathbf{b}_{i+1}^{(k)}=\underset{\mathbf{b}^{\prime}}{\arg \max }\left\{v\left(\mathbf{b}_{i}^{(k)}, \mathbf{b}^{\prime}, \mathbf{X}_{i}\right)+\left(1-\delta_{k}\right) \mathbb{E}\left\{F_{\delta_{k}}\left(\mathbf{b}^{\prime}, \mathbf{X}_{i+1}\right) \mid \mathbf{X}_{i}\right\}\right\},
$$


for $1 \leq i$. The portfolio $\mathbf{B}^{(k)}=\left\{\mathbf{b}_{i}^{(k)}\right\}$ is called the portfolio of expert $k$ with capital $S_{n}\left(\mathbf{B}^{(k)}\right)$. We combine the experts borrowing a current technique from machine learning, the exponential weighing (cf. Cesa-Bianchi and Lugosi [5]). Combine the experts as follows: let $\left\{q_{k}\right\} \geq 0$ be a probability distribution on $1 \leq$ $k$ and aggregate the experts with the wealth as described earlier by Algorithm1, so $\tilde{S}_{n}(\tilde{\mathbf{B}})=\sum_{k} q_{k} S_{n}\left(\mathbf{B}^{(k)}\right)$.

Theorem 2. Assume (i) and (ii) of Theorem 1. Choose the discount factor $\delta_{i} \downarrow 0$ as $i \rightarrow \infty$. Then, for Strategy 2 ,

$$
\lim _{n \rightarrow \infty}\left(\frac{1}{n} \log S_{n}^{*}-\frac{1}{n} \log \tilde{S}_{n}\right)=0 \text { a.s. }
$$

\section{Summary}

We considered discrete time infinite horizon growth optimal investment with several assets in stock markets with proportional transactions costs. The asset returns followed homogeneous Markov processes. Using techniques from dynamic programming and machine learning two recursive investment strategies were shown, such that, in the long run, the growth rate on trajectories were greater than or equal to the growth rate of any other investment strategy with probability 1. An important direction of our future work is to construct an empirical version of the second stationary rule, i.e., to get a data driven portfolio selection (cf. Györfi et al. 8], Györfi and Schäfer [9]) when the distribution of the market process is unknown.

\section{Proofs}

Proof of Theorem 1. Introduce the following notation: $F_{i}(\mathbf{b}, \mathbf{x})=F_{\delta_{i}}(\mathbf{b}, \mathbf{x})$. We have to show that

$$
\liminf _{n \rightarrow \infty} \frac{1}{n} \sum_{i=1}^{n}\left(g\left(\mathbf{b}_{i}^{*}, \mathbf{b}_{i+1}^{*}, \mathbf{X}_{i}, \mathbf{X}_{i+1}\right)-g\left(\mathbf{b}_{i}, \mathbf{b}_{i+1}, \mathbf{X}_{i}, \mathbf{X}_{i+1}\right)\right) \geq 0
$$

a.s. Because of the martingale difference argument in Section 2, one has

$$
\begin{aligned}
& \liminf _{n \rightarrow \infty} \frac{1}{n} \sum_{i=1}^{n}\left(g\left(\mathbf{b}_{i}^{*}, \mathbf{b}_{i+1}^{*}, \mathbf{X}_{i}, \mathbf{X}_{i+1}\right)-g\left(\mathbf{b}_{i}, \mathbf{b}_{i+1}, \mathbf{X}_{i}, \mathbf{X}_{i+1}\right)\right) \\
= & \liminf _{n \rightarrow \infty} \frac{1}{n} \sum_{i=1}^{n}\left(v\left(\mathbf{b}_{i}^{*}, \mathbf{b}_{i+1}^{*}, \mathbf{X}_{i}\right)-v\left(\mathbf{b}_{i}, \mathbf{b}_{i+1}, \mathbf{X}_{i}\right)\right)
\end{aligned}
$$

a.s. therefore we have to prove that

$$
\liminf _{n \rightarrow \infty}\left(\frac{1}{n} \sum_{i=1}^{n} v\left(\mathbf{b}_{i}^{*}, \mathbf{b}_{i+1}^{*}, \mathbf{X}_{i}\right)-\frac{1}{n} \sum_{i=1}^{n} v\left(\mathbf{b}_{i}, \mathbf{b}_{i+1}, \mathbf{X}_{i}\right)\right) \geq 0
$$


a.s. (9) implies that

$$
F_{i}\left(\mathbf{b}_{i}^{*}, \mathbf{X}_{i}\right)=v\left(\mathbf{b}_{i}^{*}, \mathbf{b}_{i+1}^{*}, \mathbf{X}_{i}\right)+\left(1-\delta_{i}\right) \mathbb{E}\left\{F_{i}\left(\mathbf{b}_{i+1}^{*}, \mathbf{X}_{i+1}\right) \mid \mathbf{b}_{i+1}^{*}, \mathbf{X}_{i}\right\},
$$

while for any portfolio $\left\{\mathbf{b}_{i}\right\}$,

$$
F_{i}\left(\mathbf{b}_{i}, \mathbf{X}_{i}\right) \geq v\left(\mathbf{b}_{i}, \mathbf{b}_{i+1}, \mathbf{X}_{i}\right)+\left(1-\delta_{i}\right) \mathbb{E}\left\{F_{i}\left(\mathbf{b}_{i+1}, \mathbf{X}_{i+1}\right) \mid \mathbf{b}_{i+1}, \mathbf{X}_{i}\right\} .
$$

Because of (12) and (13), we get that $\frac{1}{n} \sum_{i=1}^{n} v\left(\mathbf{b}_{i}^{*}, \mathbf{b}_{i+1}^{*}, \mathbf{X}_{i}\right)$

$$
\begin{aligned}
& =\frac{1}{n} \sum_{i=1}^{n}\left(F_{i}\left(\mathbf{b}_{i}^{*}, \mathbf{X}_{i}\right)-\left(1-\delta_{i}\right) \mathbb{E}\left\{F_{i}\left(\mathbf{b}_{i+1}^{*}, \mathbf{X}_{i+1}\right) \mid \mathbf{b}_{i+1}^{*}, \mathbf{X}_{i}\right\}\right) \\
& =\frac{1}{n} \sum_{i=1}^{n}\left(F_{i}\left(\mathbf{b}_{i}^{*}, \mathbf{X}_{i}\right)-\left(1-\delta_{i}\right) \mathbb{E}\left\{F_{i}\left(\mathbf{b}_{i+1}^{*}, \mathbf{X}_{i+1}\right) \mid \mathbf{X}_{1}^{i}\right\}\right)
\end{aligned}
$$

and

$$
\begin{aligned}
\frac{1}{n} \sum_{i=1}^{n} v\left(\mathbf{b}_{i}, \mathbf{b}_{i+1}, \mathbf{X}_{i}\right) & \leq \frac{1}{n} \sum_{i=1}^{n}\left(F_{i}\left(\mathbf{b}_{i}, \mathbf{X}_{i}\right)-\left(1-\delta_{i}\right) \mathbb{E}\left\{F_{i}\left(\mathbf{b}_{i+1}, \mathbf{X}_{i+1}\right) \mid \mathbf{b}_{i+1}, \mathbf{X}_{i}\right\}\right) \\
& =\frac{1}{n} \sum_{i=1}^{n}\left(F_{i}\left(\mathbf{b}_{i}, \mathbf{X}_{i}\right)-\left(1-\delta_{i}\right) \mathbb{E}\left\{F_{i}\left(\mathbf{b}_{i+1}, \mathbf{X}_{i+1}\right) \mid \mathbf{X}_{1}^{i}\right\}\right),
\end{aligned}
$$

therefore

$$
\begin{aligned}
& \frac{1}{n} \sum_{i=1}^{n} v\left(\mathbf{b}_{i}^{*}, \mathbf{b}_{i+1}^{*}, \mathbf{X}_{i}\right)-\frac{1}{n} \sum_{i=1}^{n} v\left(\mathbf{b}_{i}, \mathbf{b}_{i+1}, \mathbf{X}_{i}\right) \\
\geq & \frac{1}{n} \sum_{i=1}^{n}\left(F_{i}\left(\mathbf{b}_{i}^{*}, \mathbf{X}_{i}\right)-\left(1-\delta_{i}\right) \mathbb{E}\left\{F_{i}\left(\mathbf{b}_{i+1}^{*}, \mathbf{X}_{i+1}\right) \mid \mathbf{X}_{1}^{i}\right\}\right) \\
- & \frac{1}{n} \sum_{i=1}^{n}\left(F_{i}\left(\mathbf{b}_{i}, \mathbf{X}_{i}\right)-\left(1-\delta_{i}\right) \mathbb{E}\left\{F_{i}\left(\mathbf{b}_{i+1}, \mathbf{X}_{i+1}\right) \mid \mathbf{X}_{1}^{i}\right\}\right) .
\end{aligned}
$$

Apply the following identity

$$
\begin{aligned}
& \left(1-\delta_{i}\right) \mathbb{E}\left\{F_{i}\left(\mathbf{b}_{i+1}, \mathbf{X}_{i+1}\right) \mid \mathbf{X}_{1}^{i}\right\}-F_{i}\left(\mathbf{b}_{i}, \mathbf{X}_{i}\right) \\
= & \mathbb{E}\left\{F_{i}\left(\mathbf{b}_{i+1}, \mathbf{X}_{i+1}\right) \mid \mathbf{X}_{1}^{i}\right\}-F_{i}\left(\mathbf{b}_{i+1}, \mathbf{X}_{i+1}\right) \\
+ & F_{i}\left(\mathbf{b}_{i+1}, \mathbf{X}_{i+1}\right)-F_{i}\left(\mathbf{b}_{i}, \mathbf{X}_{i}\right) \\
- & \delta_{i} \mathbb{E}\left\{F_{i}\left(\mathbf{b}_{i+1}, \mathbf{X}_{i+1}\right) \mid \mathbf{X}_{1}^{i}\right\} \\
= & a_{i}+b_{i}+c_{i} .
\end{aligned}
$$

Because of $F_{i}(\mathbf{b}, \mathbf{x})=\max _{\mathbf{b}^{\prime}}\left\{v\left(\mathbf{b}, \mathbf{b}^{\prime}, \mathbf{x}\right)+\left(1-\delta_{i}\right) \mathbb{E}\left(F_{i}\left(\mathbf{b}^{\prime}, \mathbf{X}_{i+1}\right) \mid \mathbf{X}_{i}=\mathbf{x}\right),\right\}$ we have that $\left\|F_{i}\right\|_{\infty} \leq\|v\|_{\infty}+\left(1-\delta_{i}\right)\left\|F_{i}\right\|_{\infty}$, therefore $\left\|F_{i}\right\|_{\infty} \leq \frac{\|v\|_{\infty}}{\delta_{i}}$ (cf. Lemma 4.2.3 in Schäfer [16]). As $\left\{a_{i}\right\}$ is a sequence of martingale differences such that 
$\left|a_{i}\right| \leq 2\left\|F_{i}\right\|_{\infty} \leq \frac{2}{\delta_{i}}\|v\|_{\infty}$, therefore, because of $\sum_{n} \frac{1}{n^{2} \delta_{n}^{2}}<\infty$, the Chow Theorem implies that $\frac{1}{n} \sum_{i=1}^{n} a_{i} \rightarrow 0$ (14) a.s. (cf. Stout [18]).

Similarly to the bounding above, we have the equality

$$
F_{i}(\mathbf{b}, \mathbf{x})=\max _{\mathbf{b}^{\prime}}\left\{v\left(\mathbf{b}, \mathbf{b}^{\prime}, \mathbf{x}\right)+\left(1-\delta_{i}\right) \mathbb{E}\left(F_{i}\left(\mathbf{b}^{\prime}, \mathbf{X}_{i+1}\right) \mid \mathbf{X}_{i}=\mathbf{x}\right)\right\}
$$

and the inequality

$$
\begin{aligned}
F_{i+1}(\mathbf{b}, \mathbf{x}) & =\max _{\mathbf{b}^{\prime \prime}}\left\{v\left(\mathbf{b}, \mathbf{b}^{\prime \prime}, \mathbf{x}\right)+\left(1-\delta_{i+1}\right) \mathbb{E}\left(F_{i+1}\left(\mathbf{b}^{\prime \prime}, \mathbf{X}_{i+2}\right) \mid \mathbf{X}_{i+1}=\mathbf{x}\right)\right\} \\
& \geq v\left(\mathbf{b}, \mathbf{b}^{\prime}, \mathbf{x}\right)+\left(1-\delta_{i+1}\right) \mathbb{E}\left(F_{i+1}\left(\mathbf{b}^{\prime}, \mathbf{X}_{i+1}\right) \mid \mathbf{X}_{i}=\mathbf{x}\right)
\end{aligned}
$$

with arbitrary $\mathbf{b}^{\prime}$. Taking difference

$$
\begin{aligned}
& F_{i}(\mathbf{b}, \mathbf{x})-F_{i+1}(\mathbf{b}, \mathbf{x}) \\
\leq & \max _{\mathbf{b}^{\prime}}\left\{\left(1-\delta_{i}\right) \mathbb{E}\left(F_{i}\left(\mathbf{b}^{\prime}, \mathbf{X}_{i+1} \mid \mathbf{X}_{i}=\mathbf{x}\right)\right)\right. \\
- & \left.\left.\left(1-\delta_{i+1}\right) \mathbb{E}\left(F_{i+1}\left(\mathbf{b}^{\prime}, \mathbf{X}_{i+1}\right) \mid \mathbf{X}_{i}=\mathbf{x}\right)\right)\right\} \\
\leq & \left(1-\delta_{i}\right)\left\|F_{i}-F_{i+1}\right\|_{\infty}+\left(\delta_{i+1}-\delta_{i}\right) \max _{\mathbf{b}^{\prime}} \mathbb{E}\left(F_{i+1}\left(\mathbf{b}^{\prime}, \mathbf{X}_{i+1}\right) \mid \mathbf{X}_{i}=\mathbf{x}\right) \\
\leq & \left(1-\delta_{i}\right)\left\|F_{i}-F_{i+1}\right\|_{\infty}+\left(\delta_{i+1}-\delta_{i}\right)\left\|F_{i+1}\right\|_{\infty} .
\end{aligned}
$$

So we have $\left\|F_{i}-F_{i+1}\right\|_{\infty} \leq \frac{\delta_{i}-\delta_{i+1}}{\delta_{i}}\left\|F_{i+1}\right\|_{\infty}$. Using that $\left\|F_{i+1}\right\|_{\infty} \leq \frac{\|v\|_{\infty}}{\delta_{i+1}}$ and assumption on $\delta_{i}$ 's, we get that $\left\|F_{i}-F_{i+1}\right\|_{\infty} \leq\|v\|_{\infty} \frac{\delta_{i}-\delta_{i+1}}{\delta_{i}^{2}}$ (cf. Lemma 4.2.3 in Schäfer [16]). Concerning $\left\{b_{i}\right\}$,

$$
\begin{aligned}
& \quad\left|\frac{1}{n} \sum_{i=1}^{n} b_{i}\right|=\left|\frac{1}{n} \sum_{i=1}^{n}\left(F_{i}\left(\mathbf{b}_{i+1}, \mathbf{X}_{i+1}\right)-F_{i}\left(\mathbf{b}_{i}, \mathbf{X}_{i}\right)\right)\right| \\
& \leq\left|\frac{1}{n} \sum_{i=1}^{n}\left(F_{i}\left(\mathbf{b}_{i+1}, \mathbf{X}_{i+1}\right)-F_{i+1}\left(\mathbf{b}_{i+1}, \mathbf{X}_{i+1}\right)\right)\right| \\
& +\left|\frac{1}{n} \sum_{i=1}^{n}\left(F_{i+1}\left(\mathbf{b}_{i+1}, \mathbf{X}_{i+1}\right)-F_{i}\left(\mathbf{b}_{i}, \mathbf{X}_{i}\right)\right)\right| \\
& \leq \frac{1}{n} \sum_{i=1}^{n}\left\|F_{i}-F_{i+1}\right\|_{\infty}+\left|\frac{1}{n}\left(F_{n+1}\left(\mathbf{b}_{n+1}, \mathbf{X}_{n+1}\right)-F_{1}\left(\mathbf{b}_{1}, \mathbf{X}_{1}\right)\right)\right| \\
& \leq \frac{1}{n} \sum_{i=1}^{n}\left\|F_{i}-F_{i+1}\right\|_{\infty}+\frac{\left\|F_{n+1}\right\|_{\infty}+\left\|F_{1}\right\|_{\infty}}{n} \\
& \leq\|v\|_{\infty} \frac{1}{n} \sum_{i=1}^{n} \frac{\left|\delta_{i+1}-\delta_{i}\right|}{\delta_{i+1}^{2}}+\|v\|_{\infty} \frac{1 / \delta_{n+1}+1 / \delta_{1}}{n} \rightarrow 0
\end{aligned}
$$

by conditions. Concerning the proof of (11) what is left to show that

$$
\limsup _{n \rightarrow \infty} \frac{1}{n} \sum_{i=1}^{n} \delta_{i}\left(\mathbb{E}\left\{F_{i}\left(\mathbf{b}_{i+1}^{*}, \mathbf{X}_{i+1}\right) \mid \mathbf{X}_{1}^{i}\right\}-\mathbb{E}\left\{F_{i}\left(\mathbf{b}_{i+1}, \mathbf{X}_{i+1}\right) \mid \mathbf{X}_{1}^{i}\right\}\right) \leq 0
$$


a.s. The definition of $F_{i}$ implies that

$$
\begin{aligned}
& F_{i}\left(\mathbf{b}_{i+1}^{*}, \mathbf{X}_{i+1}\right)-F_{i}\left(\mathbf{b}_{i+1}, \mathbf{X}_{i+1}\right) \\
= & \max _{\mathbf{b}^{\prime}}\left\{v\left(\mathbf{b}_{i+1}^{*}, \mathbf{b}^{\prime}, \mathbf{X}_{i+1}\right)+\left(1-\delta_{i}\right) \mathbb{E}\left\{F_{i}\left(\mathbf{b}^{\prime}, \mathbf{X}_{i+2}\right) \mid \mathbf{X}_{i+1}\right\}\right\} \\
- & \max _{\mathbf{b}^{\prime \prime}}\left\{v\left(\mathbf{b}_{i+1}, \mathbf{b}^{\prime \prime}, \mathbf{X}_{i+1}\right)+\left(1-\delta_{i}\right) \mathbb{E}\left\{F_{i}\left(\mathbf{b}^{\prime \prime}, \mathbf{X}_{i+2}\right) \mid \mathbf{X}_{i+1}\right\}\right\} \\
\leq & \max _{\mathbf{b}^{\prime}}\left\{v\left(\mathbf{b}_{i+1}^{*}, \mathbf{b}^{\prime}, \mathbf{X}_{i+1}\right)+\left(1-\delta_{i}\right) \mathbb{E}\left\{F_{i}\left(\mathbf{b}^{\prime}, \mathbf{X}_{i+2}\right) \mid \mathbf{X}_{i+1}\right\}\right. \\
& \left.-v\left(\mathbf{b}_{i+1}, \mathbf{b}^{\prime}, \mathbf{X}_{i+1}\right)-\left(1-\delta_{i}\right) \mathbb{E}\left\{F_{i}\left(\mathbf{b}^{\prime}, \mathbf{X}_{i+2}\right) \mid \mathbf{X}_{i+1}\right\}\right\} \\
\leq & \max _{\mathbf{b}^{\prime}}\left\{v\left(\mathbf{b}_{i+1}^{*}, \mathbf{b}^{\prime}, \mathbf{X}_{i+1}\right)-v\left(\mathbf{b}_{i+1}, \mathbf{b}^{\prime}, \mathbf{X}_{i+1}\right)\right\} \\
\leq & 2\|v\|_{\infty},
\end{aligned}
$$

therefore

$$
\frac{1}{n} \sum_{i=1}^{n} \delta_{i} \mathbb{E}\left\{F_{i}\left(\mathbf{b}_{i+1}^{*}, \mathbf{X}_{i+1}\right)-F_{i}\left(\mathbf{b}_{i+1}, \mathbf{X}_{i+1}\right) \mid \mathbf{X}_{1}^{i}\right\} \leq \frac{2\|v\|_{\infty}}{n} \sum_{i=1}^{n} \delta_{i} \rightarrow 0 .
$$

(6), (14) and (15) imply (11).

\section{Proof of Theorem 2}

Theorem 1 implies that $\liminf \inf _{n \rightarrow \infty}\left(\frac{1}{n} \log S_{n}^{*}-\frac{1}{n} \log \tilde{S}_{n}\right) \geq 0$ a.s.. We have to show that

$$
\liminf _{n \rightarrow \infty}\left(\frac{1}{n} \log \tilde{S}_{n}-\frac{1}{n} \log S_{n}^{*}\right) \geq 0
$$

a.s. By definition, $\frac{1}{n} \log \tilde{S}_{n}=\frac{1}{n} \log \sum_{k=1}^{\infty} q_{k} S_{n}\left(\mathbf{B}^{(k)}\right) \geq \frac{1}{n} \log \sup _{k} q_{k} S_{n}\left(\mathbf{B}^{(k)}\right)=$ $\sup _{k}\left(\frac{\log q_{k}}{n}+\frac{1}{n} \log S_{n}\left(\mathbf{B}^{(k)}\right)\right)$, therefore (16) follows from the following:

$\liminf _{n \rightarrow \infty}\left(\sup _{k}\left(\frac{\log q_{k}}{n}+\frac{1}{n} \sum_{i=1}^{n} g\left(\mathbf{b}_{i}^{(k)}, \mathbf{b}_{i+1}^{(k)}, \mathbf{X}_{i}, \mathbf{X}_{i+1}\right)\right)\right.$ $\left.-\frac{1}{n} \sum_{i=1}^{n} g\left(\mathbf{b}_{i}^{*}, \mathbf{b}_{i+1}^{*}, \mathbf{X}_{i}, \mathbf{X}_{i+1}\right)\right) \geq 0$ a.s. which is equivalent to

$$
\liminf _{n \rightarrow \infty} \sup _{k}\left(\frac{\log q_{k}}{n}+\frac{1}{n} \sum_{i=1}^{n}\left(v\left(\mathbf{b}_{i}^{(k)}, \mathbf{b}_{i+1}^{(k)}, \mathbf{X}_{i}\right)-v\left(\mathbf{b}_{i}^{*}, \mathbf{b}_{i+1}^{*}, \mathbf{X}_{i}\right)\right)\right) \geq 0
$$

a.s. (10) implies that

$$
F_{k}\left(\mathbf{b}_{i}^{(k)}, \mathbf{X}_{i}\right)=v\left(\mathbf{b}_{i}^{(k)}, \mathbf{b}_{i+1}^{(k)}, \mathbf{X}_{i}\right)+\left(1-\delta_{k}\right) \mathbb{E}\left\{F_{k}\left(\mathbf{b}_{i+1}^{(k)}, \mathbf{X}_{i+1}\right) \mid \mathbf{b}_{i+1}^{(k)}, \mathbf{X}_{i}\right\}
$$

while for any portfolio $\left\{\mathbf{b}_{i}\right\}$,

$$
F_{k}\left(\mathbf{b}_{i}, \mathbf{X}_{i}\right) \geq v\left(\mathbf{b}_{i}, \mathbf{b}_{i+1}, \mathbf{X}_{i}\right)+\left(1-\delta_{k}\right) \mathbb{E}\left\{F_{k}\left(\mathbf{b}_{i+1}, \mathbf{X}_{i+1}\right) \mid \mathbf{b}_{i+1}, \mathbf{X}_{i}\right\},
$$

thus for the portfolio $\left\{\mathbf{b}_{i}^{*}\right\}$

$$
F_{k}\left(\mathbf{b}_{i}^{*}, \mathbf{X}_{i}\right) \geq v\left(\mathbf{b}_{i}^{*}, \mathbf{b}_{i+1}^{*}, \mathbf{X}_{i}\right)+\left(1-\delta_{k}\right) \mathbb{E}\left\{F_{k}\left(\mathbf{b}_{i+1}^{*}, \mathbf{X}_{i+1}\right) \mid \mathbf{b}_{i+1}^{*}, \mathbf{X}_{i}\right\} \text {. }
$$


Because of (18) and (19), we get that $\frac{1}{n} \sum_{i=1}^{n} v\left(\mathbf{b}_{i}^{*}, \mathbf{b}_{i+1}^{*}, \mathbf{X}_{i}\right) \leq$

$$
\begin{aligned}
& \leq \frac{1}{n} \sum_{i=1}^{n}\left(F_{k}\left(\mathbf{b}_{i}^{*}, \mathbf{X}_{i}\right)-\left(1-\delta_{k}\right) \mathbb{E}\left\{F_{k}\left(\mathbf{b}_{i+1}^{*}, \mathbf{X}_{i+1}\right) \mid \mathbf{b}_{i+1}^{*}, \mathbf{X}_{i}\right\}\right) \\
& =\frac{1}{n} \sum_{i=1}^{n}\left(F_{k}\left(\mathbf{b}_{i}^{*}, \mathbf{X}_{i}\right)-\left(1-\delta_{k}\right) \mathbb{E}\left\{F_{k}\left(\mathbf{b}_{i+1}^{*}, \mathbf{X}_{i+1}\right) \mid \mathbf{X}_{1}^{i}\right\}\right)
\end{aligned}
$$

and $\frac{1}{n} \sum_{i=1}^{n} v\left(\mathbf{b}_{i}^{(k)}, \mathbf{b}_{i+1}^{(k)}, \mathbf{X}_{i}\right)=$

$$
\begin{aligned}
& =\frac{1}{n} \sum_{i=1}^{n}\left(F_{k}\left(\mathbf{b}_{i}^{(k)}, \mathbf{X}_{i}\right)-\left(1-\delta_{k}\right) \mathbb{E}\left\{F_{k}\left(\mathbf{b}_{i+1}^{(k)}, \mathbf{X}_{i+1}\right) \mid \mathbf{b}_{i+1}^{(k)}, \mathbf{X}_{i}\right\}\right) \\
& =\frac{1}{n} \sum_{i=1}^{n}\left(F_{k}\left(\mathbf{b}_{i}^{(k)}, \mathbf{X}_{i}\right)-\left(1-\delta_{k}\right) \mathbb{E}\left\{F_{k}\left(\mathbf{b}_{i+1}^{(k)}, \mathbf{X}_{i+1}\right) \mid \mathbf{X}_{1}^{i}\right\}\right),
\end{aligned}
$$

therefore

$$
\begin{aligned}
& \frac{1}{n} \sum_{i=1}^{n} v\left(\mathbf{b}_{i}^{(k)}, \mathbf{b}_{i+1}^{(k)}, \mathbf{X}_{i}\right)-\frac{1}{n} \sum_{i=1}^{n} v\left(\mathbf{b}_{i}^{*}, \mathbf{b}_{i+1}^{*}, \mathbf{X}_{i}\right) \\
\geq & \frac{1}{n} \sum_{i=1}^{n}\left(F_{k}\left(\mathbf{b}_{i}^{(k)}, \mathbf{X}_{i}\right)-\left(1-\delta_{k}\right) \mathbb{E}\left\{F_{k}\left(\mathbf{b}_{i+1}^{(k)}, \mathbf{X}_{i+1}\right) \mid \mathbf{X}_{1}^{i}\right\}\right) \\
- & \frac{1}{n} \sum_{i=1}^{n}\left(F_{k}\left(\mathbf{b}_{i}^{*}, \mathbf{X}_{i}\right)-\left(1-\delta_{k}\right) \mathbb{E}\left\{F_{k}\left(\mathbf{b}_{i+1}^{*}, \mathbf{X}_{i+1}\right) \mid \mathbf{X}_{1}^{i}\right\}\right) .
\end{aligned}
$$

Apply the following identity

$$
\begin{aligned}
& \left(1-\delta_{k}\right) \mathbb{E}\left\{F_{k}\left(\mathbf{b}_{i+1}, \mathbf{X}_{i+1}\right) \mid \mathbf{X}_{1}^{i}\right\}-F_{k}\left(\mathbf{b}_{i}, \mathbf{X}_{i}\right) \\
= & \mathbb{E}\left\{F_{k}\left(\mathbf{b}_{i+1}, \mathbf{X}_{i+1}\right) \mid \mathbf{X}_{1}^{i}\right\}-F_{k}\left(\mathbf{b}_{i+1}, \mathbf{X}_{i+1}\right)+F_{k}\left(\mathbf{b}_{i+1}, \mathbf{X}_{i+1}\right)-F_{k}\left(\mathbf{b}_{i}, \mathbf{X}_{i}\right) \\
- & \delta_{k} \mathbb{E}\left\{F_{k}\left(\mathbf{b}_{i+1}, \mathbf{X}_{i+1}\right) \mid \mathbf{X}_{1}^{i}\right\} \\
= & a_{i}+b_{i}+c_{i} .
\end{aligned}
$$

Similarly to the proof of Theorem 1 the averages of $a_{i}$ 's and $b_{i}$ 's tend to zero a.s., so concerning (17) we have that, with probability one,

$$
\begin{aligned}
& \liminf _{n \rightarrow \infty} \sup _{k}\left(\frac{\log q_{k}}{n}+\frac{1}{n} \sum_{i=1}^{n}\left(v\left(\mathbf{b}_{i}^{(k)}, \mathbf{b}_{i+1}^{(k)}, \mathbf{X}_{i}\right)-v\left(\mathbf{b}_{i}^{*}, \mathbf{b}_{i+1}^{*}, \mathbf{X}_{i}\right)\right)\right) \\
\geq & \sup _{k} \liminf _{n \rightarrow \infty}\left(\frac{\log q_{k}}{n}+\frac{1}{n} \sum_{i=1}^{n}\left(v\left(\mathbf{b}_{i}^{(k)}, \mathbf{b}_{i+1}^{(k)}, \mathbf{X}_{i}\right)-v\left(\mathbf{b}_{i}^{*}, \mathbf{b}_{i+1}^{*}, \mathbf{X}_{i}\right)\right)\right) \\
= & \sup _{k} \liminf _{n \rightarrow \infty} \frac{1}{n} \sum_{i=1}^{n}\left(v\left(\mathbf{b}_{i}^{(k)}, \mathbf{b}_{i+1}^{(k)}, \mathbf{X}_{i}\right)-v\left(\mathbf{b}_{i}^{*}, \mathbf{b}_{i+1}^{*}, \mathbf{X}_{i}\right)\right) \\
= & \sup _{k} \liminf _{n \rightarrow \infty} \frac{\delta_{k}}{n} \sum_{i=1}^{n}\left(\mathbb{E}\left\{F_{k}\left(\mathbf{b}_{i+1}^{(k)}, \mathbf{X}_{i+1}\right) \mid \mathbf{X}_{1}^{i}\right\}-\mathbb{E}\left\{F_{k}\left(\mathbf{b}_{i+1}^{*}, \mathbf{X}_{i+1}\right) \mid \mathbf{X}_{1}^{i}\right\}\right) .
\end{aligned}
$$


The problem left is to show that the last term is non negative a.s. Using the definition of $F_{k}$

$$
\begin{aligned}
& F_{k}\left(\mathbf{b}_{i+1}^{(k)}, \mathbf{X}_{i+1}\right)-F_{k}\left(\mathbf{b}_{i+1}^{*}, \mathbf{X}_{i+1}\right) \\
= & \max _{\mathbf{b}^{\prime}}\left\{v\left(\mathbf{b}_{i+1}^{(k)}, \mathbf{b}^{\prime}, \mathbf{X}_{i+1}\right)+\left(1-\delta_{k}\right) \mathbb{E}\left\{F_{k}\left(\mathbf{b}^{\prime}, \mathbf{X}_{i+2}\right) \mid \mathbf{X}_{i+1}\right\}\right\} \\
- & \max _{\mathbf{b}^{\prime \prime}}\left\{v\left(\mathbf{b}_{i+1}^{*}, \mathbf{b}^{\prime \prime}, \mathbf{X}_{i+1}\right)+\left(1-\delta_{k}\right) \mathbb{E}\left\{F_{k}\left(\mathbf{b}^{\prime \prime}, \mathbf{X}_{i+2}\right) \mid \mathbf{X}_{i+1}\right\}\right\} \\
= & \max _{\mathbf{b}^{\prime}} \min _{\mathbf{b}^{\prime \prime}}\left\{\left\{v\left(\mathbf{b}_{i+1}^{(k)}, \mathbf{b}^{\prime}, \mathbf{X}_{i+1}\right)+\left(1-\delta_{k}\right) \mathbb{E}\left\{F_{k}\left(\mathbf{b}^{\prime}, \mathbf{X}_{i+2}\right) \mid \mathbf{X}_{i+1}\right\}\right\}\right. \\
& \left.-\left\{v\left(\mathbf{b}_{i+1}^{*}, \mathbf{b}^{\prime \prime}, \mathbf{X}_{i+1}\right)+\left(1-\delta_{k}\right) \mathbb{E}\left\{F_{k}\left(\mathbf{b}^{\prime \prime}, \mathbf{X}_{i+2}\right) \mid \mathbf{X}_{i+1}\right\}\right\}\right\} \\
\geq & \min _{\mathbf{b}^{\prime \prime}}\left\{\left\{v\left(\mathbf{b}_{i+1}^{(k)}, \mathbf{b}^{\prime \prime}, \mathbf{X}_{i+1}\right)+\left(1-\delta_{k}\right) \mathbb{E}\left\{F_{k}\left(\mathbf{b}^{\prime \prime}, \mathbf{X}_{i+2}\right) \mid \mathbf{X}_{i+1}\right\}\right\}\right. \\
& \left.-\left\{v\left(\mathbf{b}_{i+1}^{*}, \mathbf{b}^{\prime \prime}, \mathbf{X}_{i+1}\right)+\left(1-\delta_{k}\right) \mathbb{E}\left\{F_{k}\left(\mathbf{b}^{\prime \prime}, \mathbf{X}_{i+2}\right) \mid \mathbf{X}_{i+1}\right\}\right\}\right\} \\
= & \min _{\mathbf{b}^{\prime \prime}}\left\{v\left(\mathbf{b}_{i+1}^{(k)}, \mathbf{b}^{\prime \prime}, \mathbf{X}_{i+1}\right)-v\left(\mathbf{b}_{i+1}^{*}, \mathbf{b}^{\prime \prime}, \mathbf{X}_{i+1}\right)\right\} \\
\geq & -2\|v\|_{\infty},
\end{aligned}
$$

therefore

$$
\begin{aligned}
\sup _{k} \liminf _{n \rightarrow \infty} \frac{\delta_{k}}{n} \sum_{i=1}^{n} \mathbb{E}\left\{F_{k}\left(\mathbf{b}_{i+1}^{(k)}, \mathbf{X}_{i+1}\right)-F_{k}\left(\mathbf{b}_{i+1}^{*}, \mathbf{X}_{i+1}\right) \mid \mathbf{X}_{1}^{i}\right\} & \geq \sup _{k} \delta_{k}\left(-2\|v\|_{\infty}\right) \\
& =0
\end{aligned}
$$

a.s., and (17) is proved.

\section{References}

[1] Akien, M., Sulem, A., Taksar, M.I.: Dynamic Optimization of Long-term Growth Rate for a Portfolio with Transaction Costs and Logaritmic Utility. Mathematical Finance 11, 153-188 (2001)

[2] Algoet, P., Cover, T.: Asymptotic Optimality Asymptotic Equipartition Properties of Log-optimum Investments. Annals of Probability 16, 876-898 (1988)

[3] Arapostathis, A., Borkar, V.S., Fernandez-Gaucherand, E., Ghosh, M.K., Marcus, S.I.: Discrete-time Controlled Markov Processes with Average Cost Criterion: a Survey. SIAM J. Control Optimization 31, 282-344 (1993)

[4] Bobryk, R.V., Stettner, L.: Discrete Time Portfolio Selection with Proportional Transaction Costs. Probability and Mathematical Statistics 19, 235-248 (1999)

[5] Cesa-Bianchi, N., Lugosi, G.: Prediction, Learning, and Games. Cambridge University Press, Cambridge (2006)

[6] Davis, M.H.A., Norman, A.R.: Portfolio Selection with Transaction Costs. Mathematics of Operations Research 15, 676-713 (1990)

[7] Fernholz, E.R.: Stochastic Portfolio Theory. Springer, New York (2000)

[8] Györfi, L., Lugosi, G., Udina, F.: Nonparametric Kernel-based Sequential Investment Strategies. Mathematical Finance 16, 337-357 (2006)

[9] Györfi, L., Schäfer, D.: Nonparametric Prediction. In: Suykens, J.A.K., Horváth, G., Basu, S., Micchelli, C., Vandevalle, J. (eds.) Advances in Learning Theory: Methods, Models and Applications, pp. 339-354. IOS Press, Amsterdam (2003) 
[10] Györfi, L., Ottucsák, G.: Empirical log-optimal portfolio selections: a survey, manuscript (2007), http://www.szit.bme.hu/oti/portfolio/articles/tgyorfi.pdf

[11] Hernández-Lerma, L., Lasserre, J.B.: Discrete-Time Markov Control Processes: Basic Optimality Criteria. Springer, New York (1996)

[12] Iyengar, G.: Discrete Time Growth Optimal Investment with Costs. Working Paper (2002), http://www.columbia.edu/gi10/Papers/stochastic.pdf

[13] Iyengar, G., Cover, T.: Growth Optimal Investment in Horse Race Markets with Costs. IEEE Transactions on Information Theory 46, 2675-2683 (2000)

[14] Lasserre, J.B.: Sample-path Average Optimality for Markov Control Processes. IEEE Transactions on Automatic Control 44, 1966-1971 (1999)

[15] Palczewski, J., Stettner, L.: Optimisation of Portfolio Growth Rate on the Market with Fixed Plus Proportional Transaction Cost. CIS to Appear a Special Issue Dedicated to Prof. T. Duncan (2006)

[16] Schäfer, D.: Nonparametric Estimation for Finantial Investment under LogUtility. PhD Dissertation, Mathematical Institute, University Stuttgart. Shaker Verlag, Aachen (2002)

[17] Shreve, S.E., Soner, H.M.: Optimal Investment and Consumption with Transaction Costs. Annals of Applied Probability 4, 609-692 (1994)

[18] Stout, W.F.: Almost Sure Convergence. Academic Press, New York (1974)

[19] Taksar, M., Klass, M., Assaf, D.: A Diffusion Model for Optimal Portfolio Selection in the Presence of Brokerage Fees. Mathematics of Operations Research 13, 277 294 (1988)

[20] Vega-Amaya, O.: Sample-path Average Optimality of Markov Control Processes with Strictly Unbounded Costs. Applicationes Mathematicae 26, 363-381 (1999) 